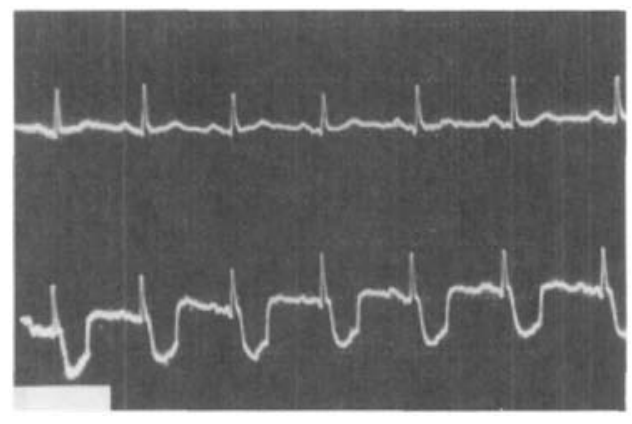

FIGURE Normal ECG pattem, during operation from line power (top). Abnormal pattem, during operation from battery (bottom).

immediately restored the ECG pattern to normal. The abnormal ECG pattern was reproduced when the monitor was plugged back into the original socket, thereby restoring operation from the battery.

Our Model 741 monitor includes a red indicator which is supposed to light when the battery voltage drops below $11.2 \mathrm{~V}$ (the normal battery output is $12.0 \mathrm{~V}$ ). This indicator did not light during the event described above.

Since encountering this problem we have become aware that a similar problem was reported to occur with a Mennen model 742 ECG monitor.' This monitor was noted to display inaccurate heart rate and spurious wave forms resembling ventricular tachycardia and other cardiac arrhythmias when operating under low battery conditions. This occurred when the monitor's battery voltage dropped below $9.5 \mathrm{~V}$. The red low-battery indicator did activate, in contrast to our experience.

The Health Devices report indicates that there are five Mennen series 700 portable ECG monitors with optional battery power (Models 740, 741, 742, 743 and 744). The report recommends that hospitals with Mennen 700 series portable monitors should contact the Manager of Technical Services, Mennen Medical at 716-759-6921 to obtain warning labels free of charge for the low battery indicator. These labels state: "Red LED indicates low battery. Return to AC power." The Health Devices report further suggets that an additional label should be attached to series 700 monitors: "Warning: Low battery operation may result in erroneous displays."

It is clearly essential that anaesthetists who use a monitor with a built-in tendency to produce an abnormal wave form in some circumstances must be completely aware of this possibility, in order to prevent unnecessary and potentially dangerous treatment being initiated in response to an abnormal ECG pattern. Our experience also serves as a reminder of another important basic premise: treat the patient, not the monitor.

N.V. Palkar, MB, BS, MD (Anaesth)

H. Moseley, FFARCs (London)

Department of Anaesthesia

Queen Elizabeth Hospital

Martindales Road

St. Michael, Barbados (W.I.)

REFERENCE

1 Mennen Series 70 Portable Monitor. Health Devices $1985 ; 14 ; 134-5$

\section{Contralateral Horner's Syndrome following stellate ganglion block}

To the Editor:

We would like to report an unusual complication of a stellate ganglion block performed on a 63-yearold patient for the treatment of a reflex sympathetic dystrophy.

A contralateral Horner's Syndrome occurred after the seventh in a series of 15 left stellate blocks. The technique used was identical to the previous six blocks. The stellate ganglion blocks were performed with the patient supine, using an anterior approach with the anterior tubercle of $\mathrm{C} 6$ as a bony landmark.' A $3.75 \mathrm{~cm} 22$-gauge needle and 7-8 cc of 0.25 per cent plain bupivacaine were used to perform the blocks. With this technique, the patient rapidly developed a left Horner's syndrome following each block.

Following the seventh block, the patient developed a right-sided ptosis and meiosis, which subsequently became a complete right Homer's syndrome. The patient had no evidence of subarachnoid block, hoarsenes or neurologic deficits in either arm. Subsequent blocks were followed by ipsilateral Homer's syndromes.

Warrick reported one case of a contralateral Horner's syndrome from a stellate ganglion block, 
which occurred during treatment of Menière's disease. ${ }^{2}$ A "large volume" of procaine had been used. The author attributed this complication to medial angulation of the needle and to the large volume of local anaesthetic injected. In our case, the volume of local anaethetic used was only $7-8 \mathrm{cc}$ with each block. The needle was inserted perpendicular to the frontal plane, using the anterior tubercle of $\mathrm{C} 6$ as a landmark, making it unlikely that the needle was angled medially.

Perhaps there was medial spread of the anaesthetic solution to the contralateral side, whether deep or superficial to the prevertebral fascia. Murphy states that injection deep to the prevertebral fascia may spread the local anaesthetic posteriorly and laterally to the fascia, involving some or all of the roots of the brachial plexus. ${ }^{1}$ Our patient did not develop any neurologic changes in the distribution of the brachial plexus.

The possibility of some variation in the anatomy of the stellate ganglion, with communication between the two sides must also be considered. This would allow local anaesthetic to track across to the contralateral side. If this was true, however, one would expect a bilateral stellate ganglion block, rather than only on the contralateral side and the repeated blocks would produce the same results.

The mechanism of production of a contralateral Horner's syndrome after a stellate block remains poorly explained and its occurrence is rare. Nevertheless, this case report illustrates the importance of good monitoring, follow-up and vigilance in the performance of seemingly routine repeated simple procedures. Unexpected results can occur and must be diagnosed and dealt with appropriately.

Gregory Allen, MD

Benoit Samson, MD, FRCPC

Department of Anaesthesia

Ottawa General Hospital

501 Smyth Road

Ottawa, Ontario

\section{REFERENCES}

1 Murphy, TM. Treatment of chronic pain in anesthesia. Miller, R.D. (ed.), Churchill Livingstone, 1981, pp. 1472-4.

2 Warrick, JW. Stellate ganglion block in the treatment of Menière's disease and in the symptomatic relief of tinnitus. Br J Anaesth 1969; 41: 699-702.

\section{Capnography and air embolism}

To the Editor:

A recent report ${ }^{1}$ by Symons and Leaver describes a case of air embolism resulting in cardiovascular collapse. The authors state that the capnogram quantitates both the severity of the pathophysiological event and the effectiveness of the treatment. The first sign of embolism was the fall in end-tidal carbon dioxide $\left(\mathrm{ET}-\mathrm{CO}_{2}\right)$ concentration from approximately four to one per cent, preceding by about 45 seconds a decrease in arterial pressure and a change in Doppler sound. We would like to make the following remarks:

1 The tracing in the figure does not show the pattern which is characteristic for pulmonary embolism. $^{2-4}$ The fall in ET- $\mathrm{CO}_{2}$ concentration occurring in the first six expirations is probably caused by air embolism. Thereafter the further fall in ET- $\mathrm{CO}_{2}$ concentration is likely to be caused by the change in cardiac output illustrated by the decrease in systemic arterial blood pressure.

Changes in cardiac output are well known to result in changes in expiratory carbon dioxide concentration. $^{5}$ We therefore believe that the tracing shows a combined effect: pulmonary embolism and change in cardiac output. The capnogram in this case does not provide a quantitative measurement of the primary pathophysiological event.

Illustration of both effects occurring separately are shown in the two figures. Figure 1 is a recording of a typical washout curve: a rapid fall in $\mathrm{ET}-\mathrm{CO}_{2}$ concentration with a gradual return to the preexisting level. The pulmonary artery pressure (PAP) increases at the same time, as is expected in the case of pulmonary embolism. ${ }^{4.6}$ There are no changes in blood pressure, central venous pressure and heart rate. This is a typical case of air embolism without systemic haemodynamic consequences. Figure 2 shows a recording with a fall and rise in ET- $\mathrm{CO}_{2}$ concentration caused by a change in cardiac output.

2 The change in Doppler ultrasound occurred $45 \mathrm{sec}$ after the decrease in ET- $\mathrm{CO}_{2}$ concentration, at the same time as the fall in blood pressure. Most likely this change in Doppler sound was not caused by air in the heart but by the 